\title{
Thin-film silicon solar cells with efficient periodic light trapping texture
}

Christian Haase, and Helmut Stiebig

Citation: Appl. Phys. Lett. 91, 061116 (2007);

View online: https://doi.org/10.1063/1.2768882

View Table of Contents: http://aip.scitation.org/toc/apl/91/6

Published by the American Institute of Physics

\section{Articles you may be interested in}

Light trapping properties of pyramidally textured surfaces

Journal of Applied Physics 62, 243 (1987); 10.1063/1.339189

$19.8 \%$ efficient "honeycomb" textured multicrystalline and $24.4 \%$ monocrystalline silicon solar cells Applied Physics Letters 73, 1991 (1998); 10.1063/1.122345

Light trapping in thin-film silicon solar cells with integrated diffraction grating Journal of Applied Physics 106, 074901 (2009); 10.1063/1.3232236

Surface plasmon enhanced silicon solar cells

Journal of Applied Physics 101, 093105 (2007); 10.1063/1.2734885

The effect of front $\mathrm{ZnO}$ :Al surface texture and optical transparency on efficient light trapping in silicon thin-film solar cells

Journal of Applied Physics 101, 074903 (2007); 10.1063/1.2715554

Photonic crystal enhanced light-trapping in thin film solar cells

Journal of Applied Physics 103, 093102 (2008); 10.1063/1.2908212

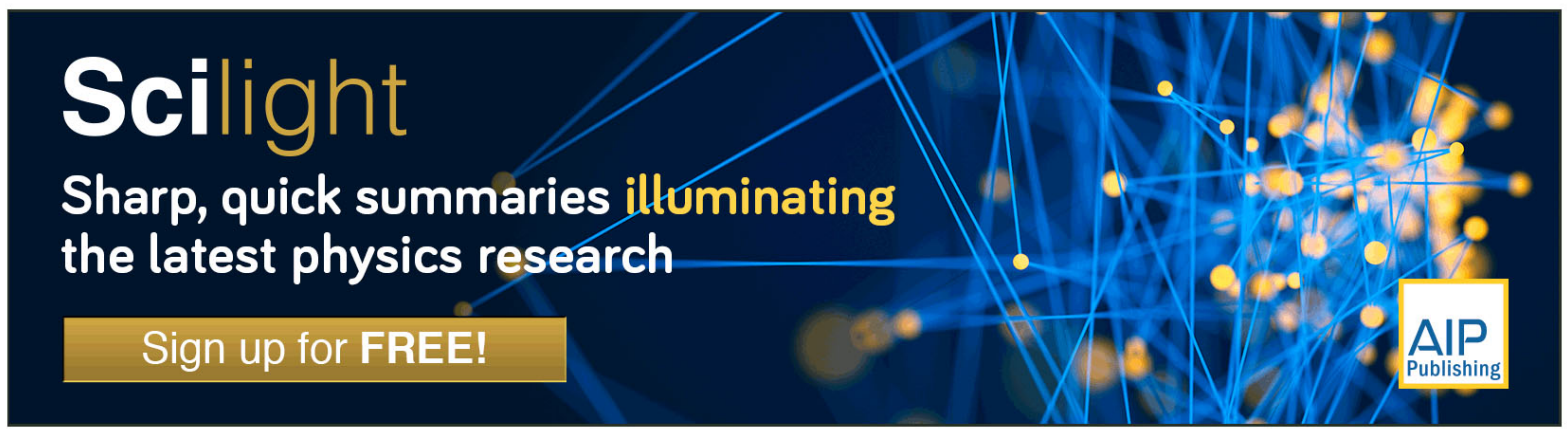




\title{
Thin-film silicon solar cells with efficient periodic light trapping texture
}

\author{
Christian Haase ${ }^{a)}$ and Helmut Stiebig \\ Institute of Photovoltaics, Forschungszentrum Juelich, D-52425 Juelich, Germany
}

(Received 11 May 2007; accepted 16 July 2007; published online 8 August 2007)

\begin{abstract}
For solar cells based on thin-film microcrystalline $(\mu \mathrm{c}-\mathrm{Si}: \mathrm{H})$ or amorphous silicon $(a-\mathrm{Si}: \mathrm{H})$ with absorber layers in the micrometer range, highly effective light trapping and an optimal incoupling of the entire sun spectrum are essential. To investigate and optimize both effects the wave propagation in thin-film silicon solar cells is modeled in three dimensions (3D) solving the Maxwell equations rigorously. A periodic nanostructured texture is investigated as an alternative to the common randomly rough texture. Inverted 3D pyramids with a periodicity of $850 \mathrm{~nm}$ and structure height of $400 \mathrm{~nm}$ show promising high quantum efficiencies close to the Tiedje limit. (C) 2007 American Institute of Physics. [DOI: 10.1063/1.2768882]
\end{abstract}

The interaction principle of light waves incident on a surface with a nanostructure in the wavelength range ${ }^{1}$ is a key question in the development of optical technologies, such as solar cells, ${ }^{2}$ light-emitting diodes, ${ }^{3}$ photonic crystals, ${ }^{4}$ and surface plasmon metal devices. ${ }^{5}$ Efficient thinfilm solar cells based on microcrystalline silicon $(\mu \mathrm{c}-\mathrm{Si}: \mathrm{H})$ or amorphous silicon $(a-\mathrm{Si}: \mathrm{H})$ with an absorber layer in the micrometer range require effective light trapping and an optimal incoupling of the entire sun spectrum. The established approach to achieve this is the application of randomly textured transparent conductive oxides (TCOs). ${ }^{6-11}$ Therefore, a postdeposition etching step to roughen the front TCO [zinc oxide $(\mathrm{ZnO})]$ layer or a naturally grown rough $\mathrm{TCO}$ is applied, ${ }^{10-12}$ with lateral feature sizes between $1-2 \mu \mathrm{m}$ and 400-800 nm, respectively. The working principle of the nanotextured substrate is neither completely understood nor optimized yet. Previous investigations of light trapping in thin-film devices have been conducted with often misleading far field measurements. ${ }^{13,14}$ However, the question regarding the optimized lateral feature size, vertical height, resulting surface angle, and shape of the texture is still unsolved but essential to reach high energy conversion efficiencies. Optical simulations based on the finite integration technique (CST MICROWAVE STUDIOß) are a valuable approach to analyze the light propagation in thin-film devices with nanotextured interfaces by solving the Maxwell equations rigorously in three dimensions (3D). ${ }^{15,16}$ It allows us to investigate the near-field optics of an $\sim 2 \mu \mathrm{m}$ thick multilayer system and consider near-field phenomena, such as optically confined modes. Therefore, the optical properties of $\mu \mathrm{c}-\mathrm{Si}: \mathrm{H}$ cells with periodically textured substrates and numerous texture designs are studied by numerical modeling using the optical constants of $\mu \mathrm{c}-\mathrm{Si}: \mathrm{H}$ determined by optical characterization of individual layers. ${ }^{17}$

The diffraction of the light incident on the grating coupler leads to a prolonged absorption path within the solar cell; because with increasing diffraction order $k$ the absorption path increases $[\sin (\theta+\psi)=k \lambda / P n] . \lambda$ denotes the vacuum wavelength, $\theta$ the angle of diffraction, $\psi$ the angle of incidence, $P$ the period size of the grating coupler, and $n$ denotes the refractive index of the material in which the transmitted, diffracted light propagates. When the diffraction angle exceeds the escape cone, the light can be totally

${ }^{a)}$ FAX: 49-2461-613735; electronic mail: c.haase@fz-juelich.de trapped within the device. This is valid for normal incidence $\psi=0$ of the incoming radiation. First studies have investigated a $\mu \mathrm{c}-\mathrm{Si}: \mathrm{H}$ diode with an integrated line grating ${ }^{18-20}$ to understand the influence of the basic texture parameters: lateral structure size (period size $P$ ) and vertical structure size (structure height $h$ ). For $P=0.7-1.2 \mu \mathrm{m}$ and $h=300-$ $400 \mathrm{~nm}$ improved quantum efficiencies in the long wavelength range and improved short circuit currents were calculated. For wavelength $\lambda<500 \mathrm{~nm}$ an insignificant improvement of the light incoupling was found for rectangular shaped line gratings. These tendencies were also observed for prototypes of cells with rectangular shaped transparent line gratings. ${ }^{19}$ A change of the texture from a rectangular shaped line grating to a multistep line grating resulted in an improved incoupling of blue light (300-500 nm). Thus light incoupling depends strongly on the shape of the grating. ${ }^{21}$ When this two-dimensional (2D) multistep line grating is transformed to a 3D multistep pyramid texture the red light trapping is strongly enhanced. ${ }^{21}$ The additional space dimension of the structure results in a prolonged absorption path. To avoid interference effects originating from the size and number of steps in the following square based pyramids with flat interfaces and an inverted orientation concerning the light incidence are used (Fig. 1). Flat interfaces and a 3D

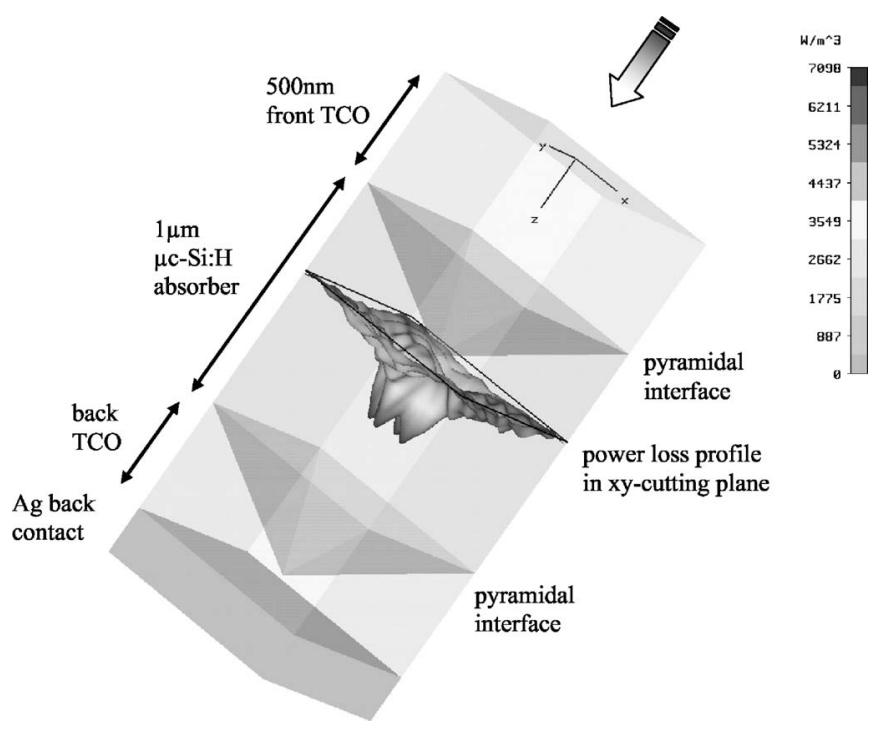

FIG. 1. Scheme of simulation unit cell-power loss profile of 3D pyramid texture for illumination with $\lambda=400 \mathrm{~nm}$. 


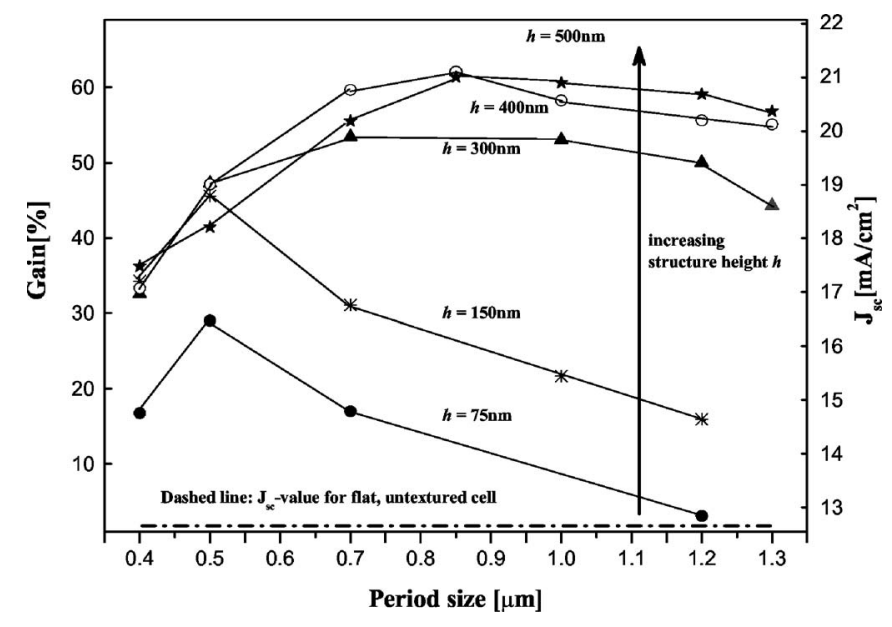

FIG. 2. $J_{\mathrm{sc}}$ gain of a pyramid texture in comparison to an untextured cell as a function of the period size.

texture also match closer to the commercial approach. In this study a variation of $P$ and $h$ of the 3D pyramid is performed and the results are plotted as a function of the resulting opening angle $\alpha$.

The solar cell is described by a unit cell consisting of a $1 \mu \mathrm{m}$ thick $\mu \mathrm{c}-\mathrm{Si}: \mathrm{H}$ absorber with a single pyramid structure (Fig. 1). For $300 \mathrm{~nm}<\lambda<1100 \mathrm{~nm}$ the unit cell is illuminated with a circular polarized plane wave, which enters under normal incidence. As an example, a power loss profile for illumination with $\lambda=400 \mathrm{~nm}$ is plotted in a $x y$-cutting plane in the middle of the absorber layer. The symmetric peaks represent "modes" that are determined by the optical confinement of the cell design. The absorbed power is calculated and the external quantum efficiency (EQE), i.e., the probability of the generation of an electron-hole pair through a photon, can be derived by $\operatorname{EQE}(\lambda)=$ power (absorbed)/ power (incident). The optical simulations do not consider internal electrical effects such as carrier recombination or other electrical losses. The calculated $J_{\mathrm{sc}}$ values are the maximum possible short circuit current density. An EQE value below one implies that the incident radiation has not been completely absorbed within the photovoltaic active region. It can be reflected at the front side of the cell, absorbed in the $\mathrm{TCO}_{\text {front }}$ or $\mathrm{TCO}_{\text {back }}$ layer or it might have escaped from the cell after multiple reflections within the cell. Especially for a $\lambda>620 \mathrm{~nm}$, the absorption coefficient $a$ multiplied with the layer thickness $d$ is $a d<1$. Thus light has to be multiple reflected within the cell until it is absorbed. Considering the sun spectrum and the calculated EQE, $J_{\mathrm{sc}}$ can be calculated. More details regarding the modeling tool can be seen elsewhere. ${ }^{19}$ Figure 2 shows the calculated $J_{\text {sc }}$ of cells with textured substrates in comparison to a flat, untextured structure as a function of $P$ (right axis). The following parameters were used within the simulation: $P=400-1300 \mathrm{~nm}, h$ $=75-500 \mathrm{~nm}$, and the resulting pyramid opening angle $\alpha$ $=44^{\circ}-166^{\circ}$. The $J_{\mathrm{sc}}$ curves for $h=300,400$, and $500 \mathrm{~nm}$ show similar high current values with a broad maximum for $P$ around $700-1000 \mathrm{~nm}$. For lower periods $J_{\mathrm{sc}}$ strongly decreases and a weaker decrease in $J_{\mathrm{sc}}$ is observed for $P$ above $1000 \mathrm{~nm}$. The highest values were calculated for $P$ $=850 \mathrm{~nm}$ and $h=400 \mathrm{~nm}$. The short circuit current of $J_{\mathrm{sc}}$ $=21.03 \mathrm{~mA} / \mathrm{cm}^{2}$ shows a gain of $\sim+62 \%$ compared to an untextured device with an absorber thickness $=1 \mu \mathrm{m}$ (left axis). For $h=75$ and $150 \mathrm{~nm}$ and $P>500 \mathrm{~nm} J_{\text {sc }}$ is strongly

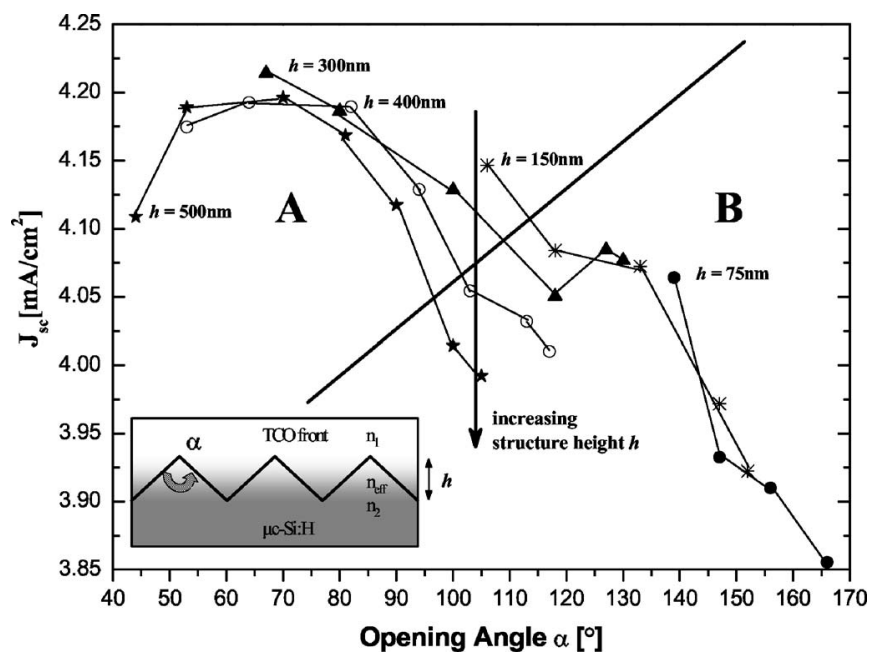

FIG. 3. Blue response as a function of the opening angle $\alpha$ and the pyramid structure height $h$. The arrow indicates the increasing structure height for a fixed opening angle.

reduced due to the insufficient light trapping properties of these structures.

To analyze the effect of light trapping, light incoupling, and their superposition two parts of the sun spectrum are discussed in the following (spec. $1=300-500 \mathrm{~nm}$ and spec. $2=700-1100 \mathrm{~nm}$ ). Due to the small penetration depth for blue light, a light trapping effect is negligible for spec. 1. Although the total amount of $J_{\mathrm{sc}}$ generated by spec. 1 is only in the range of $4 \mathrm{~mA} / \mathrm{cm}^{2}$, it is the spectral part that allows for identifying the principle of light incoupling into the device. The calculated $J_{\text {sc }}$ using spec. 1 is plotted in isolines (same structure heights) as a function of $\alpha$ (Fig. 3). The highest values are reached for small $\alpha\left(53^{\circ}<\alpha<82^{\circ}\right)$ and $h=300-500 \mathrm{~nm}$, denoted as region A. Steep structures result in a strong blue response as observed in concepts with naturally rough grown $\mathrm{ZnO} .^{22}$ For a fixed $\alpha$ of $\sim 100^{\circ}$ the blue response decreases with increasing lateral structure sizes, as indicated by the arrow in Fig. 3. With increasing lateral structure size the optical properties of the interface change from improved light incoupling due to effective refractive index matching to the regime, where bulk optical properties prevail. For light with shorter wavelength (spec. 1) the light incoupling properties of the cell mainly determines $J_{\mathrm{sc}}$. According to the effective refractive index grading approach a complex geometrical structure with materials of different refractive indices can be approximately described by an effective refractive index $n_{\text {eff. }}$. An interface reflection is in general reduced by a continuous change in refractive index. The inset of Fig. 3 shows, simplified to $2 \mathrm{D}$, the principle change in refractive index that is introduced by the pyramids. Large $P$ leads to a discontinuous lateral intermixing of the two refractive indices and the plane wave is thus reflected by a more abrupt change in $n$. Region $\mathrm{B}$ has in relation to the incident waves too large period sizes that inhibit a good light incoupling.

The front TCO also leads to absorption losses. To enable a lateral current flow the TCO is comprised by a $500 \mathrm{~nm}$ thick flat layer and a textured region with inverted pyramids. Caused by this approach, the absorption losses within the $\mathrm{TCO}_{\text {front }}$ contact should be more pronounced for TCOs with higher $h$. The front contact layer absorbs at least 5\% and even up to $50 \%$ for $\lambda$ between 300 and $400 \mathrm{~nm}$. The integra- 


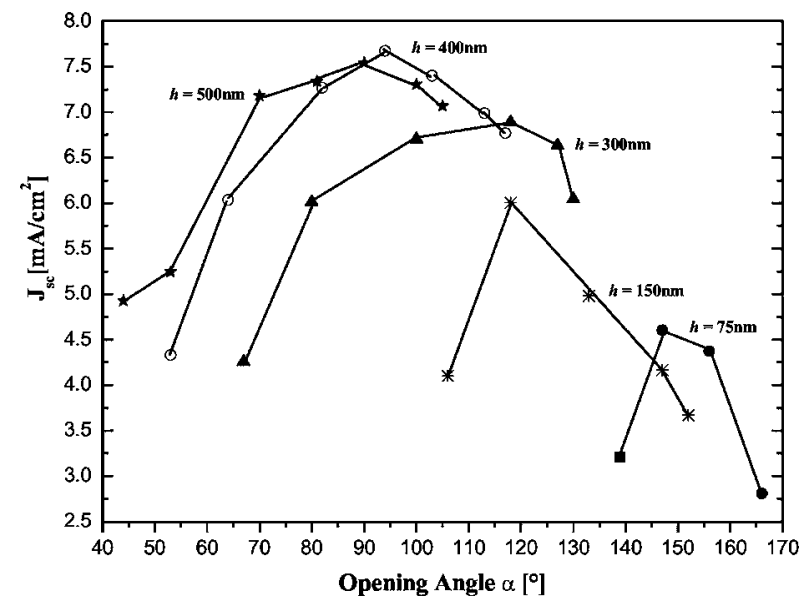

FIG. 4. Red response as a function of the opening angle $\alpha$ and the pyramid structure height $h$.

tion of a taller TCO pyramid with $h=300-500 \mathrm{~nm}$ (for $P$ $=0.7 \mu \mathrm{m})$ would result in a decrease of the $J_{\mathrm{sc}}$ by about $\sim-0.1 \mathrm{~mA} / \mathrm{cm}^{2}$ in comparison to a small pyramid with $h$ $=75 \mathrm{~nm}$ and $P=0.7 \mu \mathrm{m}$. However, this increased parasitic absorption is overcompensated by the improved incoupling properties of the optimized texture. A total gain in $J_{\text {sc }}$ of $\sim+0.4 \mathrm{~mA} / \mathrm{cm}^{2}$ is found.

In Fig. 4 the red response is plotted with regard to $\alpha$. The curves for $h=300,400$, and $500 \mathrm{~nm}$ have a similar shape. The $J_{\mathrm{sc}}$ maximum is shifted to smaller angles for higher $h$. For $h=75,300,400$, and $500 \mathrm{~nm}$ it is at around $147^{\circ}-156^{\circ}$, $100^{\circ}-127^{\circ}, 90^{\circ}-103^{\circ}$, and $81^{\circ}-100^{\circ}$, respectively. A broad distribution of high values of $J_{\mathrm{sc}}=6.8-7.7 \mathrm{~mA} / \mathrm{cm}^{2}$ is generally found for angles between $70^{\circ}$ and $113^{\circ}$ and $h=400$ and $500 \mathrm{~nm}$. The isolines for the same structure height have, for increasing $\alpha$, a similar decrease in $J_{\mathrm{sc}}$ as the resulting angles of diffraction are getting too small. The light is not diffracted ideally into the silicon layer. The chance for an escape of the light from the structure is high and the desired waveguide effect is not efficient. For decreasing $\alpha$ no efficient light trapping occurs as the propagation of higher order modes is not supported. Structures with $h=75$ and $150 \mathrm{~nm}$ are in general too small to induce a sufficient diffraction for long wavelength light and consequently their light trapping potential is low.

The presented data correspond to a back contact that has

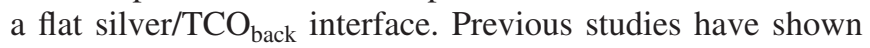
that this back contact system exhibits a higher red light trapping potential than a conformal interface, especially for small periods. ${ }^{21}$ The optimum for incoupling and light trapping occurs at different angles. This results in a relative broad maximum for the choice of texture parameters with good optical properties which are also influenced by the scattering and reflection properties of the back contact. Within the simulations an ideal metal back contact was considered. In conclusion, light trapping can be to a certain extent insensitive to slight changes in $\alpha, h$, and $P$. In contrast to conventional gratings and macro-optical devices the investigated subwavelength gratings/structures perform more consistently over a broader range of wavelengths than conventional gratings.

The theoretical maximum cell current density for $\lambda$ $=650-1100 \mathrm{~nm}$ was determined by Tiedje et al. ${ }^{23}$ (using the idealized Yablonovitch light trapping limit) to be
$16.8 \mathrm{~mA} / \mathrm{cm}^{2}$. Vanecek et $a .^{24}$ calculated a limit of $14.0 \mathrm{~mA} / \mathrm{cm}^{2}$ considering nearly idealized conditions such as antireflection (AR) coatings, high $\mathrm{TCO}_{\text {front }}$ transparencies, Lambertian scattering, and high back contact reflectivity. The cell current determined for the presented periodic structures (see Fig. 2, $P=0.85 \mu \mathrm{m}$ and $h=400 \mathrm{~nm}$ ) for $\lambda=650$ $1100 \mathrm{~nm}$ is $J_{\mathrm{sc}}=10.0 \mathrm{~mA} / \mathrm{cm}^{2}$. However, this structure exhibits strong $\mathrm{TCO}_{\text {front }}$ and $\mathrm{TCO}_{\text {back }}$ absorption losses in the range of $5.8 \mathrm{~mA} / \mathrm{cm}^{2}$. Increasing the optical transparency of the TCO layers has in conclusion a great potential to substantially increase the cell current. Within our simulations we do not consider a glass sheet. Thus the entering plane wave experiences an abrupt change from $n_{\text {air }}$ to $n_{\mathrm{TCO}}$ instead of a smoother change in refractive index with the glass sheet present. Therefore, the overall reflectivity of our unit cell is approximately 5\% too high. We estimate the maximum current that can be reached by the presented periodic light trapping structures to be $J_{\mathrm{sc}}^{\max }=1.05\left(10.0+5.8 \mathrm{~mA} / \mathrm{cm}^{2}\right)$ $=16.6 \mathrm{~mA} / \mathrm{cm}^{2}$. This is close to the Tiedje limit and above the value determined by Vanecek. A fine tuning of $P, h$, and the structure shape in addition to the use of AR coatings could lead to even higher values.

The good agreement between these simulations, investigated prototypes with periodic line grating texture, and our experience with various random texture designs proves the reliability and versatility of the performed Maxwell simulations. This numerical 3D analysis gives clear design criteria for efficient light incoupling and light trapping textures. For the investigated solar cell design the periodic structure has to be in the range of $P=0.7-1.2 \mu \mathrm{m}, h=300-500 \mathrm{~nm}$, and pyramid opening angles $\alpha=60^{\circ}-130^{\circ}$, respectively.

${ }^{1}$ E. Yablonovitch, Phys. Rev. Lett. 58, 2059 (1987).

${ }^{2}$ M. Green, Third Generation Photovoltaics (Springer, Berlin, Germany, 2003).

${ }^{3}$ J. Wierer, M. Krames, J. Epler, N. Gardner, M. Craford, J. Wendt, J. Simmons, and M. Sigalas, Appl. Phys. Lett. 84, 3885 (2004).

${ }^{4}$ E. Yablonovitch, Sci. Am. 285, 47 (2001).

${ }^{5}$ W. Barnes, A. Dereux, and T. Ebbesen, Nature (London) 424, 824 (2003).

${ }^{6}$ S. Guha, J. Yang, and A. Banerjee, Prog. Photovoltaics 8, 141 (2000).

${ }^{7}$ Y. Mai, S. Klein, R. Carius, H. Stiebig, L. Houben, X. Geng, and F. Finger, J. Non-Cryst. Solids 352, 1859 (2006).

${ }^{8}$ S. Hegedus, Phys. Rev. A 14, 393 (2006).

${ }^{9}$ K. Yamamoto, M. Yoshimi, Y. Tawada, Y. Okamoto, and A. Nakajima, J. Non-Cryst. Solids 266-269, 1082 (2000).

${ }^{10}$ M. Kondo, T. Matsui, Y. Nasuno, H. Sonobe, and S. Shimizu, Thin Solid Films 501, 243 (2006).

${ }^{11}$ O. Kluth, G. Schöpe, J. Hüpkes, C. Agashe, J. Müller, and B. Rech, Thin Solid Films 442, 80 (2003).

${ }^{12}$ A. Shah, P. Torres, R. Tscharner, N. Wyrsch, and H. Keppner, Science 285, 692 (1999).

${ }^{13}$ J. Krc, F. Smole, and M. Topic, Prog. Photovoltaics 11, 429 (2003).

${ }^{14}$ J. Springer, A. Poruba, and M. Vanecek, J. Appl. Phys. 96, 5329 (2004).

${ }^{15}$ T. Weiland, AEU 31, 116 (1977).

${ }^{16} \mathrm{H}$. Wolter and T. Weiland, AIP Conf. Proc. 297, 74 (1994).

${ }^{17}$ K. H. Jun, R. Carius, and H. Stiebig, Phys. Rev. B 66, 115301 (2002).

${ }^{18} \mathrm{R}$. Morf and J. Gobrecht, Proceedings of the 10th QUANTSOL, 1998.

${ }^{19}$ C. Haase and H. Stiebig, Prog. Photovoltaics 14, 629 (2006).

${ }^{20}$ H. Stiebig, N. Senoussaoui, C. Zahren, C. Haase, and J. Müller, Prog. Photovoltaics 14, 13 (2006).

${ }^{21}$ C. Haase and H. Stiebig, Proc. SPIE 6197, 32 (2006).

${ }^{22}$ A. Shah, H. Schade, M. Vanecek, J. Meier, and E. Vallat-Sauvain, Prog. Photovoltaics 12, 113 (2004).

${ }^{23}$ T. Tiedje, E. Yablonovitch, G. D. Cody, and B. G. Brooks, IEEE Trans. Electron Devices 31, 711 (1984).

${ }^{24}$ M. Vanecek, J. Springer, A. Poruba, O. Kluth, T. Repmann, B. Rech, N. Wyrsch, J. Meier, and A. Shah, Proceedings of the Third WCPEC, Osaka, Japan (IEEE, New York, 2003), Vol. B, p. 1527. 